\section{Ausbruch aus der Marktnische?}

\section{Eine Rekordzahl von über tausend Ausstellern auf der biofach 98, der welt- weił größten Fachmesse für Naturkost und Naturwaren, manifestiert einen positiven Umsatztrend. Steht daher dem Einzelhandel der Durchbruch der öko- logischen Sortimente in breitere Marktsegmente bevor?}

Von Helmut Hagemann

$\mathrm{D}$ e grünen Branchen haben sich am Markt etabliert, ihre Umsätze wachsen teilweise mit zweistelligen Zuwachsraten - und dies während der konventionelle Einzelhandel in einer anhaltenden Krise steckt. Jenseits der Trends zeigt jedoch der Blick auf die absoluten Marktdaten, daß die ökologisch anspruchsvollen Produktangebote noch immer nur einen minimalen Anteil an den Gesamtumsätzen der jeweiligen Branchen repräsentieren.

Das Beispiel der erfolgreichsten ökologischen Warengruppe - Naturkost - illustriert die Position der ökologischen Anbieter: Der Anteil von Produkten aus ökologischem Anbau lag 1997 bei 1,2 bis 1,5 Prozent des Gesamtumsatzes mit Lebensmitteln in Deutschland. Dies entsprach einem Naturkostumsatz von 2,9 bis 4,1 Mrd. DM über alle Vertriebswege. Davon vertrieben die Naturkostfachgeschäfte Lebensmittel im Wert von 1,2 Mrd. DM und die Direktvermarkter im Wert von 0,6 bis 1,0 Mrd. DM. Der konventionelle Lebensmitteleinzelhandel machte, bei steigender Tendenz, einen Naturkostumsatz in Höhe von 0,5 bis 1,0 Mrd. DM (1). Dem wird Naturkost selbst bei stetigen Zuwachsraten noch lange eine Nische darstellen. Eine qualitative Veränderung deutet sich allerdings dadurch an, daß die konventionellen Einzelhandelsketten immer stärker in diesen Markt eintreten, einen wachsenden Teil der Produktion aufkaufen und mit ihren Preisgestaltungsmöglichkeiten den ÖkoPionieren Konkurrenz machen.

Andere Märkte weisen ähnliche Strukturen auf. Die vom Bundesverband Naturkost Naturwaren beobachteten Naturkosmetika-Anbieter tätigen Umsatzzahlen in der geschätzten Größenordnung von 80 Mio. DM und repräsentieren damit rund ein halbes Prozent des gesamten Kosmetikumsatzes. Die kleine Zahl der die NaturtextilienBranche dominierenden Versandhäuser schreibt nach Beobachtungen von Insidern Jahresumsätze von wenigen hundert Millionen DM. Die rund hundert ökologischen Einrichtungshäuser setzten ebenfalls weniger als ein Prozent des Umsatzes konventioneller Händler um. Ähnliches gilt für die Angebote der weniger als hundert explizit umwelt- und sozialverträglichen Reiseveranstalter und -vermittler (2). Auch die Umsatzgrößen von fair gehandelten Produkten, Öko-Waschmitteln oder naturnahen Gartenprodukten bewegen sich jeweils im Bereich von fünf bis zehn Promille. Allerdings haben viele konventionelle Einzelhandelsunternehmen inzwischen fest Fuß gefaßt im Geschäft mit umweltfreundlichen Artikeln auf verschiedenen Anspruchsniveaus. So werben Großversandhäuser intensiv mit entsprechenden Textil-Kollektionen, die meisten Hobby- und Baumärkte vertreiben lösemittelfreie Lacke, natürliche Bau- oder Dämmstoffe oder Torfersatzstoffe und Produkte mit dem. Blauem Engel der Jury Umweltzeichen sind weit verbreitet.

\section{Wie weiter?}

Der größte Teil der Artikel, die ein privater Haushalt nachfragt, läßt sich, dies ist die erste Quintessenz dieser Marktbeobachtungen, aus den Angeboten konsequent ökologischer Händler decken. Vom Alpaca-Pullover über das Naturholzhaus bis zur Zahnbürste sind ungezählte Öko-Produkte am deutschen Markt. Vor allem für die Produkte des täglichen Bedarfs gibt es differenzierte Sortimente.

Die zweite - vorläufige - Quintessenz lautet, daß die Hersteller von anspruchsvollen, nachweislich konsequent ökologischen Waren selbst bei überdurchschnittlichen Wachstumsraten auf absehbare Zeit nicht mehr als einen geringfügigen Beitrag zur Ökologisierung des Konsums der privaten Haushalte leisten können. Dies liegt am allgemein hohen Preisniveau, an der nicht flächendeckenden Präsenz der ökologischen Einzelhändler und am Zeitbedarf für den Ausbau der Produktionskapazitäten.

Wenn die Perspektive der angestammten Naturkost- und Naturwarenläden auch noch langfristig auf ein Nischendasein verweist, so sind der Ökologisierung der Angebote des konventionel- len Einzelhandels damit jedoch keine grundsätzlichen, unüberwindbaren Grenzen gesetzt. Neben einzelnen Artikeln, etwa im Lebensmittelbereich, die mit anerkannten, konsequenten Umweltzertifikaten ausgezeichnet sind, existieren solche Artikel, deren Kennzeichnung für ökologisch weniger konsequente Anspruchsniveaus (etwa im Textilbereich oder bei Elektrogroßgeräten) steht, oder deren Umweltbilanz gegenüber anderen Produkten günstiger ist, ohne daß dies ausgewiesen ist (etwa wiederaufladbare Batterien). Die Angebote des Einzelhandels unterscheiden sich hinsichtlich ihrer Umweltbilanz demnach nicht nur nach ökologisch oder nicht-ökologisch, sondern graduell über mehrere Stufen.

Eine Ökologisierungsstrategie für den Einzelhandel darf sich daher nicht darauf beschränken, nur Nischenprodukte zu fördern. Vielmehr müssen vermehrte Anstrengungen darauf verwandt werden, die Spielräume zur Ökologisierung aller Angebote zu nutzen und den Kunden den Vorteil der entsprechenden Produktentwicklungen zu kommunizieren. Für die Nischenmärkte, die Qualitätssegmente und die Niedrigpreismärkte sind jeweils angepaßte Ökologisierungsstrategien erforderlich. Dieser Weg kann dazu führen, daß mit graduellen Verbesserungen an Massenprodukten über die Summe der großen Umsatzzahlen ökologische Entlastungswirkungen erzielt werden, die hinter denen an den Nischenmärkten nicht zurïckstehen. Eine wichtige Voraussetzung für eine solche, breit ansetzende Strategie ist aber die Existenz einer etablierten Öko-Nische, die die Orientierungsmaßstäbe für ,best practice“ setzt.

\section{Anmerkungen}

(1) Vgl. Bundesverband Noturkost Naturwaren (BNN): Trendbericht Naturkost, Hürth 1998. Wegen der Ungenauigkeit der Umsatzgrößen beschreiben viele Aussagen nur Größenordnungen.

(2) Forum anders reisen, mündliche Mitteilung vom 11.03.1998.

\section{Der Autor}

Dr. Helmut Hagemann ist Leiter des Forschungsfeldes ökologischer Konsum im Institut für ökologische Wirtschafisforschung, Regionalbüro Nordrhein-Westfalen.

Kontakt: Völklinger Str. 9, 42285 Wuppertal, Tel. 0202/80530, Fax 0202/83402,

E-mail: mailbox@ioew.w.eunet.de 
(c) 20I0 Authors; licensee IÖW and oekom verlag. This is an article distributed under the terms of the Creative Commons Attribution Non-Commercial No Derivates License (http://creativecommons.org/licenses/by-nc-nd/3.o/), which permits unrestricted use, distribution, and reproduction in any medium, provided the original work is properly cited. 